\title{
Naïve Normativity: The Social Foundation of Moral Cognition
}

ABSTRACT: To answer tantalizing questions such as whether animals are moral or how morality evolved, I propose starting with a somewhat less fraught question: do animals have normative cognition? Recent psychological research suggests that normative thinking, or ought-thought, begins early in human development. Recent philosophical research suggests that folk psychology is grounded in normative thought. Recent primatology research finds evidence of sophisticated cultural and social learning capacities in great apes. Drawing on these three literatures, I argue that the human variety of social cognition and moral cognition encompass the same cognitive capacities and that the nonhuman great apes may also be normative beings. To make this argument, I develop an account of animal social norms that shares key properties with Cristina Bicchieri's account of social norms but which lowers the cognitive requirements for having a social norm. I propose a set of four early developing prerequisites implicated in social cognition that make up what I call naive normativity: (I) the ability to identify agents, (2) sensitivity to in-grouplout-group differences, (3) the capacity for social learning of group traditions, and (4) responsiveness to appropriateness. I review the ape cognition literature and present preliminary empirical evidence supporting the existence of social norms and naive normativity in great apes. While there is more empirical work to be done, I hope to have offered a framework for studying normativity in other species, and I conclude that we should be open to the possibility that normative cognition is yet another ancient cognitive endowment that is not human-unique.

KEYWORDS: moral psychology, evolution of morality, animal cognition, folk psychology, social norms

\section{Introduction}

Like language and upright walking, morality is universal across human cultures. Is morality also unique to the human species? Recent research on nonhuman animals challenges the idea that adult human beings are the only moral folk. For example, great apes and cetaceans engage in behaviors similar to those that moral

\footnotetext{
This article has taken some time to come to fruition, and I am grateful to the many, many philosophers and scientists who read drafts, listened to talks, and raised questions that helped me develop the ideas that emerge here. While I wish I could name all those who were part of the process, I must extend particular thanks to Laura Schlingloff and Richard Moore, who early on in the process challenged me to develop an account of social norms, and Dorit Bar-On, who helped me refine the concepts.
} 
foundations theory finds across cultures in human ethical practice (Vincent, Ring, and Andrews 2018). Recent philosophical research has raised challenges to the view that moral participation is unique to human beings, with arguments that animals can track moral truths (Rowlands 20I2), or even be moral agents (Monsó 20I5). While others disagree, claiming that moral agency requires metacognitive capacities that only human beings enjoy (e.g., Kitcher 20I I; Korsgaard 20I 8), the debate between these two camps may be largely terminological and nonsubstantive (Fitzpatrick 2017). To move the debate ahead, I propose a different approach to investigating the evolution of morality.

Having moral agency and acting in accordance with the moral facts describes one way of participating in a normative world. Having social norms and normative cognition describes another, more fundamental way. Normative cognition is required on all familiar approaches to the practice of morality as it includes considerations about what one ought to do regardless of the source of authority, and whether or not the end is categorical, instrumental, self-justifying, or objectively valuable. While normative cognition may not be sufficient for moral participation, it is necessary: to take a moral stance toward the world, whether one is calculating likely outcomes or following laws, is to have a kind of ought-thought. To understand better the evolution of moral practice, we can first uncover the elements of normative thinking and then examine how widespread those elements might be in the animal kingdom.

Drawing on recent work in the philosophy of mind suggesting that the way human beings understand one other is essentially regulative and normative (Andrews 20I 5; McGeer 2007, 20I5; Zawidzki 20I3), I argue that human social cognition and normative cognition are both grounded in a set of four related prerequisites implicated in social cognition that make up what I call naïve normativity: (I) the ability to identify agents, (2) sensitivity to in-group and out-group differences, (3) the capacity for social learning of group traditions, and (4) responsiveness to appropriateness. The fundamental cognitive requirement for being a social cognizer and a normative thinker is the ability to distinguish agents from nonagents. However, to have a human style of social cognition is also to be aware that these other beings belong to different social groups and that the way our group does things is, largely, the way we should do things. I show that any being who has this style of social cognition is also a participant in normative practice.

While human normative practice develops into a full-blown moral practice that permits us to engage in cognitively sophisticated behaviors such as critiquing or justifying group norms, to examine the evolution of morality, we benefit from looking at a less-developed normative practice. Just as a study of the evolution of language should not begin with an analysis of hip hop or lyric poetry, study of the evolution of morality should not begin with an analysis of moral theories. Instead, I examine the origins of ought-thought.

I offer preliminary evidence that chimpanzees are engaged in normative practice and ought-thought. Through a review of the current ape cognition literature, I show that great apes largely share a human style of social cognition, and I present preliminary empirical evidence supporting the existence of naïve normativity in 
great apes. My conclusion is that we should be open to the possibility that normative cognition is yet another ancient cognitive endowment that is not unique to human beings.

To start the discussion I offer a working account of norms. Cristina Bicchieri's (2017) social expectation account of norms has played a prominent role in philosophical discussions of norms, and is a promising place to begin. I describe a cognitively modest norm type had by human beings that plays an intermediate role between Bicchieri's two types of norms-descriptive norms and social norms. This intermediate type, which I call animal social norms, grounds my investigation of the evolution of normativity.

\section{Social Norms}

In Norms in the Wild, her influential account of social norms, Bicchieri describes social norms as group level regularities-rules-that are sustained by the psychological states of the group members themselves. Group members are motivated to follow the rules because they have a social expectation that others will follow the rules. Specifically, a social norm is a rule of behavior that individuals choose to follow because they believe two things: (I) that others in their community follow the rule and (2) that others also believe that community members ought to follow the rule (Bicchieri 20I7, 35). These two beliefs make up a requirement that individuals have a particular attitude toward normative behaviors. While the account does not include a principle that norm violators are sanctioned, the beliefs required for norms are typically accompanied by beliefs about the proper treatment of norm violators.

Bicchieri's account carries with it high cognitive demands. To have a social norm, individuals must have metacognitive access to the rules of behavior they are following. They also have to be able to attribute beliefs about those rules to other people in their community. This makes mindreading or theory of mind necessary for having a social norm. Since the capacity to attribute beliefs arises in childhood and does not appear to be widespread among other species, we might conclude that only older children or adult human beings can have social norms. That would be hasty. Instead, we can ask whether there are human practices that look like social norms but lack the mindreading aspect. That is, are there practices that human beings choose to engage in because they are the group's practices and others expect them to act in this way, without needing to explain the conformity in terms of belief in a rule?

There are a number of behavioral patterns_candidate social norms-that appear normative but do not require the capacity to represent rules as rules or the ability to attribute to others belief in the rules. Examples of these candidate social norms include greeting practices (such as hugging, shaking hands, touching the heart, bowing, or kissing one cheek, two cheeks, or three cheeks), hygienic issues (such as how and where to blow one's nose or use the toilet), talking distance norms (such as how close you may comfortably stand to talk to someone you do not know very well). Such behaviors are so ubiquitous that they dissolve into the fabric of society. They may not be noticed because that is just the way things are, 
like breathing or speaking. It may take meeting someone from outside your culture or traveling to another culture to even recognize some of one's own normative practices. This is true even when violations of these cultural practices would be extremely aversive, such as if a stranger were to stand so close to you when speaking that you could smell their breath and feel their spittle hit your face.

In addition to social norms that are all but invisible, there are social norms that are not understood by their followers. Anthropologists studying human cultures have identified causal opacity or lack of instrumentality as important aspects of social ritual. Group members engage in some practices not because they know what they are for or how they work but because that is the way they do things. Joseph Henrich (2017) gives an example of a Mapuche man who was preparing mote, a traditional corn dish: 'He showed me how you have to scoop fresh ash out of the wood stove and put it into the corn mix for soaking, before heating it. I thought that was curious, so I asked him why he mixed the wood ash in with the corn. His answer was, “It's our custom' (IO2). What the man did not know was that adding ash to the corn releases niacin, which is needed to avoid a pellagra, a fatal disease.

Such practices present like social norms in that they are required by the culture, and violations evoke sanctions. A child who made mote without ash would be corrected. However, these practices need not require mindreading others' reasons for action such that group members can explain normative behavior in terms of reasons. For one, people may be acting according to the rule without knowing that there is a rule. While in such cases one might be inclined to say that the people are implicitly forming beliefs about the rules and implicitly attributing such beliefs about following the rules to others, they still are not able to explain behavior in terms of the rules. Even when a rule is stated, they may have to check to see if it is correct. In the cases where we know that there is a rule, like 'Add ash when soaking corn', we do not need to know why there is a rule or what justifies the rule. Such observations suggest that while social norms play a causal role in producing behaviors, they still may not be accessible to the actor as explanations of behavior. Bicchieri's second condition, that people believe that others also believe that community members ought to follow the rule, requires more folk psychological machinery than necessary to predict group normative behavior.

One may object that the examples I am providing count not as social norms, but as descriptive norms. Bicchieri (2017, I9) explains descriptive norms merely as patterns of behavior with which individuals choose to conform because they believe others in their community conform, such that deviations would be costly. She offers linguistic behavior as a type of descriptive norm. Within English-speaking communities, one uses the word tree to refer to a tree. A community member who utters hutang instead of tree is not going to be a very good communicator, so they will suffer a cost, even though that cost does not take the form of a sanction.

The difference between descriptive norms and the examples described above can be seen in the differing dispositions people would have toward violators in the two cases. While individuals expect that others will conform to the descriptive norm behaviors, they are not committed to the normative claim that people ought to conform to those behaviors. For example, if it is a descriptive norm that people 
use their right hand to eat and write, a natural lefty living in a right-handed world might find it worthwhile to try to conform. Otherwise, the person would suffer environmental costs, such as being unable to use coffee mugs or spiral-ringed notebooks. On the other hand, if it is a social norm that people should use their right hand for such behaviors, additional costs would arise in the form of social sanction. While Bicchieri's account of social norms does not explicitly endorse the principle that norm violators are sanctioned, a functional description of her second condition, that people believe that others also believe that community members ought to follow the rule, can be given in terms of the disposition to sanction norm violators. That is, because we attribute beliefs to others based on their behavior, the community members will act in such a way that they believe that there are social norms, which requires more than just following the norms, as would be sufficient for descriptive norms. They must respond to norm violations to demonstrate that they are not appropriate. In other words, the only empirically available nonverbal evidence that an individual forms the belief others ought to follow some rule is the sanctioning of violations.

Practices such as greeting, hygienic issues, and how close to stand to others, as well as opaque cultural practices, do not cleanly fit into either Bicchieri's social norm or descriptive norm categories. This suggests that we need another norm type, one that is richer than her descriptive norm (because violations of these norms lead to sanctions), and leaner than her social norm (since they do not require mindreading others' beliefs about following the norm). With such an account, we can avoid setting the bar so cognitively high as to make an evolutionary analysis of sanctioning norm violations impossible.

I propose a kind of norm called animal social norms, for which there are three conditions: ( $\mathrm{I}$ ) there is a pattern of behavior demonstrated by community members; 2) individuals choose to conform to the pattern of behavior; and (3) individuals expect that community members will also conform and will sanction those who do not conform. The pattern of behavior demonstrated by community members can be taken as a rule, given that individuals choose to conform and expect others to conform. The expectation that others conform and will sanction those who do not can be taken as an attitude toward the pattern of behavior, as can sanctions in response to nonconformists. This account of animal social norms is offered in the spirit of Bicchieri's account, but it lowers the cognitive requirements for participating in social norms since it does not require the capacity to attribute mental states to others. It still requires that following of a pattern is a choice, and so it requires that normative agents are cognitively flexible. Because cognitive flexibility is widespread in the animal kingdom, this requirement does not exclude swaths of animals the way the mindreading requirement does.

Animal social norms resonate with the normative turn in folk psychology. In the next section, I show that animal social norms are integral to the normative thinking in human social cognition. Even young children demonstrate naïve normativity in their developing social cognitive capacities. 


\section{Naïve Normativity and Normative-Regulative Folk Psychology}

A recent turn of thought regarding the nature of folk psychology, or commonsense understanding of others as minded beings, has emphasized the normative and regulative nature of our social cognitive practices. Traditional theories of folk psychology describe human beings as predicting and explaining behavior by attributing beliefs and desires and appealing to a descriptively accurate theory of behavior. The normative-regulative turn, in contrast, takes our social cognitive practices to be grounded in the tasks of creating and discovering norms of behavior to coordinate, predict, explain, and understand people's actions and to include a plurality of cognitive mechanisms involved in these practices (for defenses see Andrews 20I 2, 20I 5; Maibom 2007; McGeer 2007, 201 5; Zawidzki 20I3).

Normative-regulative views share a commitment to the idea that social engagement creates expectations about how people should behave, feel, and think, and that violations of these expectations lead to restorative practices, such as explaining, justifying, and apologizing. Human beings have rich abilities in this domain because we see others as intentional agents with goals, even when we fail to consider what beliefs or desires they might have. Much work is done by seeing others as members of various social groups, and we construct and rely on generalizations about how members of those social groups should act-we use stereotypes and social norms. Of course, we also sometimes think about what others believe and desire, but we do so only against a backdrop of this richer understanding of individuals and their social relations.

The normative-regulative approach to folk psychology and animal social norms share an overlapping set of prerequisites-naïve-normativity: the ability to identify agents, sensitivity to in-group/out-group differences, capacity for social learning of group traditions, and responsiveness to appropriateness. At minimum, a folk psychologist needs to be able to distinguish agents from other things, and has to be able to learn behavioral regularities from observing behaviors. The regulative aspect of folk psychology adds to this view the idea that we are motivated to conform to the same behavioral patterns as our in-group members; their behavior shapes our behavior. The normative aspect of folk psychology introduces a responsiveness to the appropriateness or inappropriateness of a behavioral pattern; responsiveness to the appropriateness of the pattern serves as an endorsement while responsiveness to the inappropriateness of the pattern serves as a sanction.

Below, for each of the four elements, I provide evidence that they are apparent early in human children, show how they are implicated in animal social norms, and explain how they fit into the normative-regulative folk psychological framework.

\section{I Identifying Agents}

Fundamentally, social cognition involves seeing others as agents. This is both the most basic aspect of naïve-normativity and the one that requires the least amount 
of analysis. Agents, in the sense relevant here, are potential social partners who are cognitively flexible, self-propelled beings who act to achieve their goals.

The current evidence suggests that sensitivity to agency appears early in infancy. Infants expect anthropomorphized geometrical figures to engage in goal-directed behavior by twelve months (Gergely et al. I995). Sorting agents from nonagents is also an early developing capacity in human infants (Woodward I999), and it is necessary for the other three aspects of naïve normativity.

The ability to identify others as agents is a fundamental capacity on all theories of folk psychology, though this is not often made explicit. While individuals may differ on the extension of agency, with some seeing it in natural occurrences, plants, or robots, what is shared is the ability to draw a distinction between those things that are seen to act and other things that are merely moved. The account of animal social norms relies on this basic capacity because identifying movement as a pattern of behavior invokes the agency of the actors.

\subsection{Sensitivity to In-Group/Out-Group Differences}

A number of recent studies suggest that human infants quickly develop in-group preferences, and this phenomenon has been widely studied in the context of combatting racial bias. In a seminal study on looking time, newborns showed no preference between same race and other race faces, while three-month-old Caucasian infants exposed only to members of their own race preferred same-race faces over others (Kelly et al. 2005). This study suggests that there is something special about how infants perceive people, given that infants generally prefer to look at novel rather than familiar stimuli; such observations served as the basis for the now-classic habituation of looking-time method for assessing infants' cognitive capacities (Oakes 20I0). In another seminal study, six-month-old infants prefer those who speak their own language, and ten-month-olds preferentially accepted toys from a speaker using the child's native language (Kinzler, Dupoux, and Spelke 2007). Subsequent studies have built on both these findings. At seven months, infants prefer to follow the gaze of unreliable same-race adults rather than unreliable other-race adults (Xiao et al. 20I8). At eight months, infants habituate more quickly to native speakers acting prosocially than to foreign speakers acting prosocially, or either group acting antisocially (Pun et al. 20I8). At nine months, infants expect that two individuals who share the same food preferences will be affiliated, but that two individuals who differ in their preferences will interact negatively (Liberman, Kinzler, and Woodward 20I4). At twelve months, infants prefer a puppet who likes the same kind of food they do (Mahajan and Wynn 20I2). At seventeen months, infants expect adults who label themselves as part of the same minimal group (by saying 'I'm a bem' or 'I'm a tig') will help the minimal group member but not outgroup members (Jin and Baillargeon 20I7). By eighteen months, infants expect in-group preference in the distribution of resources (Bian, Sloane, and Baillargeon 2018). These studies are taken as evidence that in-group favoritism arises early in human development.

Because social norms are contextualized in relation to one's own community, a norm follower will need to distinguish in-group from out-group members. This 
ability is essential for part of the first condition for animal social norms, which is recognizing a pattern of behavior among community members. It is also essential for a folk psychologist who predicts behavior based on one's group memberships.

\subsection{Social Learning of Group Traditions}

The early ability of human infants to recognize in-group members offers opportunities for social learning, facilitating the ability to learn culturally specific behaviors. Transmission of cultural knowledge about artifacts and behaviors, from using a spoon to using language, is accomplished through processes such as imitation, apprenticeship, and facilitative teaching. By fourteen months, infants over-imitate, a form of social learning in which one imitates not just causally necessary actions, but all elements of demonstrated actions, whether they are needed or not-signifying that by this age infants have the disposition to go on in the 'right way' - the in-group way (Meltzoff I988). Over-imitation is often taken to be key to the transmission of culturally specific behaviors, since there are almost always some other ways of achieving a goal. An over-imitator wants to achieve the goal the way we do things around here, which provides a mechanism for the type of cumulative culture that many researchers take to be unique to human beings (Henrich 20I7; Tomasello I999; Richerson and Boyd 2005). Evidence that motivation to imitate serves an in-group conformity function comes from the findings that not any demonstrator will do. For example, if the demonstrator tells a story in a foreign language before performing an action, fourteen-month-olds will not over-imitate (Buttelmann et al. 2013).

Because a norm follower is motivated to conform to a community's pattern of behavior, social learning in terms of observation and imitation is essential for participating in normativity; it is my second condition for having an animal social norm-that individuals choose to conform to the pattern of behavior. By internalizing the group's behavior, a child can more easily anticipate other group members' behaviors via a simple conformity expectation. I can predict others' behavior by asking what I would do, since (most) everybody does the same thing. In addition, social learning of community behaviors also facilitates predicting in terms of the situation (such as bow in temple, not in the grocery store), emotions (such as happy to have a birthday party, angry when a toy breaks), teleology (such as ask for crayons after receiving a coloring book), and perceptual content (such as want a slice of cake after seeing it). Finally, it can help children see that there are subgroups within the society and help them learn that there are different behaviors appropriate for people in different roles (for example, parents hug and kiss you but police officers do not).

\subsection{Responsiveness to Appropriateness}

Responsiveness to appropriateness involves recognizing when a behavior is acceptable, permitted, obligatory, forbidden, and the like, and responding behaviorally or psychologically to the behavior; it is the most obviously normative of the four capacities. Sanctioning behavior is typically taken as indicative of 
responsiveness to appropriateness. Punishment is one kind of sanction. Punishments can include grand public acts, but also subtle actions such as hidden emotional responses, whispered stories to close friends, or giving the perpetrator the cold shoulder. For example, if a conversation partner steps too close, responsiveness to appropriateness may be expressed by taking a step backward. If instead you witness this interaction, responsiveness to appropriateness can be expressed by avoiding social interactions with the close talker.

Restorative justice is another kind of sanction, and it may be preferred by communities that cannot bear the costs of punishment or those that prioritize repair over punishment. Restorative justice is widespread in small-scale societies due to its many benefits: it allows stakeholders to speak and be heard, it offers compensation for losses, and it respects long-term relationships (Braithwaite 2002; Wiessner and Pupu 20I2). Punishment, on the other hand, may be costly, creating long-term feuds that inhibit cooperation and isolate individuals who might otherwise have developed into productive community members.

Responsiveness to appropriateness can also take the form of an emotional response, including guilt, shame, or embarrassment in response to one's own or a close other's inappropriate behavior, and anger, disgust, or contempt toward another whose behavior is inappropriate. While these are familiar points, it is less common to recognize that appropriateness can be demonstrated and reinforced through successful coordination of behavior. When group members anticipate that the target will engage in a particular behavior in some situation, and the target does engage in that behavior, the goals of social engagement are met, and the expectations are reinforced in the group members. But when the target does not engage in the predicted behavior, this leads to a failed social engagement. In human beings, as in great apes, this can lead to sanctions, such as chimpanzees' shunning group members (Goodall I986).

The study of children's developing responsiveness to appropriate and inappropriate behavior has largely focused on the more visible types of responses. Early research on children's moral and conventional thinking found that preschoolers express their awareness that some actions are inappropriate (Nucci and Turiel I978). More recent research finds that preschool children follow scripts and schemas in pretend play and protest when these scripts are violated (Rakoczy, Warneken, and Tomasello 2008), even though they are unable to articulate the rules (Rakoczy, Warneken, and Tomasello 2009). This suggests that children learn and countenance norms before they can identify, much less justify, the rules. Even children from four to nine years old prefer that rule violators apologize rather than give reasons for their violation (Banerjee, Bennett, and Luke 2010). This suggests that these children have a preference for restoration when a norm is violated.

Responsiveness to appropriateness is required for my third condition of animal social norms, that individuals expect community members will also conform, and will sanction those who do not conform. When individuals expect that community members will behave in a particular way, but they do not behave in that way, responsiveness to inappropriateness is exhibited by some form of sanction. Sanction involves cost, and third-party sanction (sanction in response to an act that does not directly harm the sanctioning individual) is thought to be 
required if norms are to be stable over time. Wanting others to follow norms even when violations are not directly harmful to self is indicative of an internalization of normative force, and paying a cost to sanction violators indicates that the norm is strongly held. Responsiveness to appropriateness is also implicated for folk psychologists when group members violate social norms. When an expectation fails, we may offer an explanation why, and use that explanation to anticipate future behavior. But as more and more expectations fail, the target individual might start shifting from an in-group to out-group member.

\section{Naïve Normativity in Chimpanzees}

Applying the naïve normativity framework to chimpanzees helps support the view that normative cognition and social cognition are an intertwined part of our ancient cognitive endowment. I choose chimpanzees because we have the best data on this species, not because there is reason to think that chimpanzees alone among nonhuman animals are naïvely normative.

Chimpanzees live in groups that range in size from fewer than fifty to almost two hundred individuals. Chimpanzee communities vary in size, territory range, food resources, social practices, and food-processing technologies. But all chimpanzees observed live in fission-fusion communities, such that small, unstable parties travel together in various compositions for hours to days before rejoining the group. In addition, across communities females tend to transfer between groups when they are adolescents.

Despite the challenges associated with gathering information about ape behavior and societies (including laborious field observations and small data sets from captive individuals) in the last sixty years we have gained a significant understanding of chimpanzee cognition and community. There is current evidence that chimpanzees identify agents, distinguish in-group members from out-group members, and learn the group's social traditions. There is also preliminary evidence that chimpanzees are responsive to inappropriate behavior, suggesting that apes share with human beings the foundation for moral practice.

\section{I Identifying Agents}

As I describe below, chimpanzees have the ability to identify others as agents. Note that infant chimpanzees perform like human infants on Gergely and colleagues' (I995) teleological reasoning task, suggesting that they ascribe goals to shapes that behave as agents (Uller 2004).

\subsection{Sensitivity to In-Group/Out-Group Differences}

Distinguishing in-group from out-group members can be a matter of life and death for chimpanzees. One of the longest running chimpanzee research projects is in the Mahle mountains in Tanzania, where there are a number of chimpanzee communities living in overlapping territories that vary from ten to thirty square kilometers (Nishida 20I2). 
The chimpanzee groups are antagonistic toward one another, and their patterns of antagonism are particularly interesting. Chimpanzees can stay informed about the activities of other groups by patrolling the edges of their territory. Patrolling chimpanzees sometimes cross into 'enemy territory', attacking individuals in the other group, and occasionally killing males and infants (Watts and Mitani 200I). Successful incursions result in expanded territories and increased food resources.

A particularly intense example of chimpanzee group thinking comes from the interactions between the $\mathrm{M}$ - and $\mathrm{K}$ - groups at Mahale, who were in a state of tension for years before one of the communities went extinct. Over a number of years, the six adult males in K-group disappeared one by one. The scientists inferred that M-group had killed most if not all of the males because they were all in good health, and each disappeared after an encounter between $\mathrm{K}$ - and M-groups. When only two males were left in K-group, the adult females began to migrate to M-group, and soon after the K-group was completely gone.

The primatologist Toshisada Nishida, who spent his career observing the Mahale chimpanzees, describes chimpanzee in-group thinking this way:

[C]himpanzees make a clear distinction between members and non-members of the group. The apes not only fear and loathe other groups, but they seem to have a desire to eradicate them completely. When one group becomes aware of another group nearby, their hair stands on end, they display looks of horror, they cling to their cohorts, and they experience bouts of diarrhoea. Members of the same group are occasionally treated as members of an outside group, namely 'ostracized' individuals such as former alpha males . . . the alpha male has a great influence over who is regarded as a group member and who is not . . . there is a tendency to follow the crowd, and in particular, a strong tendency for the crowd to take the alpha male's side. (Nishida 20I 2: 246-47)

Nishida's description is particularly interesting, as it demonstrates that dislike of out-group individuals is a result of thinking of them as out-group individuals. An in-group member can become an out-group member through ostracization. An infant born from an out-group male can be accepted as an in-group member, or not, based on the behavior of the alpha male. The flexibility inherent in chimpanzee group membership strongly implies that there is more going on than merely a dislike of strangers. Out-group members include known individuals.

Additional evidence of chimpanzee in-group thinking comes from the behavior of females after they immigrate to a new group. When first immigrating, females have low rank and are bullied by the resident females, but resident males tend to come to their aid (Kahlenberg et al. 2008). There is some evidence that immigrants must learn the cultural traditions of their new group after they immigrate. For example, Taï Forest females moving into a new group modified their nut-cracking behavior to conform to the behavior present in their new community. These immigrants stopped using easy-to-find wood hammers and switched to the group's practice of using harder-to find-stone hammers even though they did not serve as better tools 
for cracking nuts (Luncz and Boesch 20I4). The observation that chimpanzees will sacrifice easier access to food for social conformity with new in-group members suggests that identifying with a group and conforming to the group behavior is of significant benefit.

\subsection{Social Learning of Group Traditions}

Apes come to learn their cultural behaviors the same way human beings do, through close observation of actions and actors. While some think that apes learn cultural behaviors via a process of emulation, that is, a low-fidelity copying of the end result of observed action (Tennie, Call, and Tomasello 2006; Tomasello I999), current evidence suggests that great apes imitate the shape of a model's action, not just the outcome (Whiten et al. 2009, Byrne and Russon 1998). Diffusion studies are one way we can observe how individuals learn new behaviors. In diffusion studies with chimpanzees, a novel behavior, such as sliding open a box to access food, is seeded by demonstrating it to one individual who has been separated from the group (Whiten et al. 2007). Then the behavior is spread by either letting the individual back into the community with the apparatus, or via a daisy-chain method of letting another individual B observe $\mathrm{A}$, letting $\mathrm{C}$ observe $\mathrm{B}$, and so on. These studies find that the method demonstrated to the seeder is the method that spreads through the community. Even when another method is discovered through free play, the innovator tends to revert to the seeded method.

It seems that having a model is important for learning new behaviors, because if models are replaced by a 'ghost' who moves the object (by using transparent fishing wire, for example), the chimpanzees will not always learn the new behavior (Hopper et al. 2007). And while chimpanzees can emulate the actions of ghosts, the fidelity of their action is lower when copying the ghost than it is when copying a chimpanzee model (Hopper et al. 2008). This suggests that a model can be very important to chimpanzees, and that they are motivated to imitate the behaviors of group members, as human infants are.

Chimpanzees also appear to imitate others selectively, demonstrating learning biases. For example, wild chimpanzees are more likely to imitate dominant individuals than lower-ranking ones, even if the low-ranking chimpanzee behavior is more efficient (Kendal 20I5). Like human beings who buy the clothes they see movie stars wearing or who choose technology based on what is popular rather than what is good, chimpanzee choice also reflects the status of the individuals in their community.

Unlike human children, wild-born sanctuary chimpanzees do not tend to over-imitate obviously causally irrelevant behaviors (Horner and Whiten 2004). When a model demonstrated on an opaque puzzle box, chimpanzees copied every action, but when the box was transparent, and it was apparent how to extract the food, the chimpanzees skipped the unnecessary behavior. Children, on the other hand, tend to over-imitate actions they know are causally irrelevant, even when warned not to imitate the 'silly' actions (Lyons, Young, and Keil 2007).

It is common to interpret the failure of the sanctuary chimpanzees to over-imitate as evidence that chimpanzees are not concerned with high-fidelity imitation of 
cultural behaviors and then to use this failure to explain the relative lack of cumulative culture in chimpanzee societies (see, for example, Sterelny 20I2; Suddendorf 20I3; Tomasello I999, 20I4; Whiten et al. 2009). However, wild chimpanzees may not see human researchers as in-group members, and chimpanzees in zoos may not take graduate students clad in medical scrubs to be in-group members either. If this is so, then given the emerging consensus that over-imitation functions to help children learn cultural norms (Allen and Bickhard 20II; Rakoczy, Warneken, and Tomasello 2008) we would expect that chimpanzees might lack the motivation to over-imitate such models. Like wild chimpanzees who do not imitate the novel behavior of recent immigrants, the sanctuary chimpanzees might interpret the unfamiliar human beings as the wrong kind of model. There is evidence that chimpanzees will over-imitate in-group human beings; Tetsuro Matsuzawa, who has a lifetime research relationship with Chimpanzee $\mathrm{Ai}$, found that she over-imitated his irrelevant tool use (Myowa-Yamakoshi and Matsuzawa 2000).

The sanctuary chimpanzees may be engaged in a form of rational imitation (as in Gergely, Bekkering, and Király 2002), thinking that human beings perform the irrelevant behaviors due to some limitation. After all, to a chimpanzee, human beings are severely limited-we are unable to eat with our feet, we cannot travel on the canopy, we cannot catch and kill monkeys with our bare hands, we cannot bite open prickly fruit. If human beings are inadequate, they need to make these extra moves on the apparatus, while chimpanzees feel free to skip them.

\subsection{Responsiveness to Appropriateness}

The question that remains is whether chimpanzees are responsive to appropriateness, recognizing and responding to behaviors that are acceptable, permitted, obligatory, forbidden, and the like. The best evidence of responsiveness to appropriateness would come hand in hand with evidence of an animal social norm, since a norm violation is the sort of behavior that should elicit some sort of response indicating inappropriateness. The clearest places to find responsiveness to appropriateness are where there are established patterns of behavior and a violation of that pattern. Evidence of sanctioning, in terms of punishment, protest, shunning, retaliation, or some form of restorative justice would serve as evidence of responsiveness to appropriateness. Relatedly, we can look for evidence that chimpanzees enjoy watching transgressors sanctioned by others. We can also look for evidence of affective responses to norm violators in terms of emotional facial expressions, bodily postures, physiological measures of arousal such as hormone levels and the like, and we can examine whether abiding by norms or violating norms has an impact on social standing or reputation-social evaluation. Unfortunately, much of this research has yet to be done. The focus has primarily been on punishment understood narrowly as a visible retaliation against a transgressor.

Before reviewing the evidence, we should address a common worry that arises about animal normative behavior: how can we distinguish approving from preferring? The alpha chimpanzee might intervene in a fight because he personally 
dislikes the uproar and prefers a calm environment, not because he thinks a calm environment is best for the group or is worried about the well-being of others. A familiar way of putting this worry comes from the psychological egoist, who suggests that all human behaviors are motivated by individual desires, and hence there are only selfish behaviors and no altruistic ones. A classic response is to point out that some desires are other-directed-people act from both empathy and self-directed desires. There is quite a bit of behavioral evidence of empathy in chimpanzees, from helping another chimpanzee even when there is no benefit to self (Yamamoto, Humle, and Tanaka 2009) to consoling allies after they lose fights (Kutsukake and Castles 2004; de Waal 2009) or when they are in distress (Fraser, Stahl, and Aureli 2008). In a recent longitudinal study of empathy in chimpanzees, researchers found that, like human beings, there are stable individual differences in consolation tendencies and that those with stronger tendencies have a higher degree of social integration (Webb et al. 20I7). Continuing research on the neurological correlates of empathy across species and the modulation of consolation behavior in rats through blocking oxytocin uptake suggests that other-directed emotions are shared between human beings and other species (see Monsó and Andrews forthcoming for a review). If we can take chimpanzees' other-directed emotions as a given, then the interpretation of the following behaviors are no more open to egoist worries than are human behaviors.

Evidence for third-party physical punishment in chimpanzees is mixed. Captive studies have so far failed to find evidence of third-party punishment in experimental settings. Chimpanzee mothers in a zoo were offered the opportunity to physically punish another chimpanzee who took food from her child, but failed to do so (Jensen, Call, and Tomasello 2007; Riedl et al. 20I2). While this might look like evidence that chimpanzees do not punish, other interpretations are possible. Perhaps the mothers feared later retaliation or did not think that it was wrong for an adult to take food from an infant.

Field research and observational studies suggest that the experiments might be missing something. There is ample evidence of chimpanzee policing-physically intervening to stop fights, which is a kind of third-party punishment. Frans de Waal (I982) describes alpha males breaking up fights between lower ranking individuals, regardless of their social relationships with those individuals. These observations are supported by a recent observational study showing third-party punishment of freeloading chimpanzees in a prosocial task (Suchak et al. 20I6).

There is some evidence that captive chimpanzees appreciate it when wrongdoers are punished. In a study comparing chimpanzees and human children, researchers found that both groups will 'pay' to watch a transgressor be punished. Chimpanzees saw a human acting either prosocially (giving food to another person) or antisocially (teasing another and not giving them food). Later the actor appeared in front of the subject's enclosure alone, and a punisher appeared, expressing rage and hitting the actor. The actor and punisher moved to another part of the room, visible only after the chimpanzee subject opened a heavy door. Watching the antisocial actor be beaten was worth the effort for the chimpanzees, who opened the door more frequently when the antisocial actor was punished (Mendes et al. 20I8). While one might worry that a chimpanzee should not care 
about an out-group norm violator, it seems that human beings, too, are keen to watch out-group norm violators be punished. Recall the spectacle of Saddam Hussain's execution.

The punishment literature is based on assumptions about which behaviors may be prohibited in chimpanzee societies. As illustrated in table $\mathrm{I}$, a more promising way to examine responsiveness to appropriateness is to start by identifying candidate animal social norms-patterns of behavior that group members expect others to follow.

A candidate animal social norm is one that fulfills the first two conditions for an animal social norm: ( $\mathrm{I}$ ) there is a pattern of behavior demonstrated by community members, and (2) individuals choose to conform to the pattern of behavior. When such a pattern is identified, scientists can conduct observational or experimental studies to determine whether the third condition, that individuals expect that community members will also conform and will sanction those who do not conform, is also present. Below, I briefly describe how two of the candidate animal social norms described in table $\mathrm{I}$, infanticide avoidance and inequity aversion, may fulfill the third condition.

In chimpanzee societies, infanticide is relatively rare, happening in one community of Gombe chimpanzees only five times in forty years (Murray, Wroblewski, and Pusey 2007). Chimpanzee infants are typically tolerated by adult members of the society, and even high-ranking adult males will endure infants climbing on them and stealing tools and food. A juvenile who did the same thing would elicit aggressive responses. Males also self-handicap when playing with infants. If an adult male is aggressive toward a chimpanzee infant, even unrelated females protest with 'massive reactions'-screaming, barking, and attempting to intervene (as discussed in Rudolf von Rohr et al. 20I2; Rudolf von Rohr et al. $2015)$. Such reactions may express disapproval and serve as a sanction.

The second example, inequity aversion, offers evidence that some captive chimpanzees have developed norms regarding provisioning, such as an 'equal pay for equal work' norm (Brosnan, Schiff, and de Waal 2005). In these studies, a pair of chimpanzees is caged next to one another and given the same task. While one receives a delicious grape for the work, the other receives a less valuable cucumber slice. The cucumber receiver quickly expresses anger and then stops working, but even the grape receiver might stop working, as if in solidarity with the partner who was not receiving the same high reward.

That chimpanzees protest violations of candidate norms, whether those norms take the form of cultural traditions or expectations formed in experimental settings, is some evidence that chimpanzees demonstrate responsiveness to appropriateness. However, as this aspect of naïve normativity is the most difficult to observe, it is worth looking for convergent evidence supporting responsiveness to appropriateness. In addition to looking for visible protests and punishments, long-term studies of community responses to individuals who violate candidate animal norms might help us uncover some more subtle sanctions.

Examination of facial expressions for evidence of emotional responses to observations of norm violations is another approach. In one study researchers showed chimpanzees videos of unknown chimpanzees engaging in a variety of behaviors; some of the videos showed infanticide. Subjects looked longer at videos 
Table I. Candidate Social Norms for Chimpanzees

\begin{tabular}{|c|c|}
\hline & Candidate Animal Social Norms for Chimpanzees \\
\hline $\begin{array}{l}\text { Infanticide } \\
\text { avoidance }\end{array}$ & $\begin{array}{l}\text { Chimpanzee females protest infanticide (Rudolf von Rohr et al., 20 I 2; Rudolf } \\
\text { von Rohr et al. 20I 5). }\end{array}$ \\
\hline Treatment of infants & $\begin{array}{l}\text { Chimpanzee infants enjoy permissive parenting for the first years of life and } \\
\text { are not punished (de Waal 2OI4). }\end{array}$ \\
\hline Helping & $\begin{array}{l}\text { Male and dominant chimpanzees aid females and youth in road crossing } \\
\text { (Hockings, Anderson, and Matsuzawa 2006). Chimpanzees destroy } \\
\text { hunting snares that can injure group members (Ohashi and Matsuzawa } \\
\text { 20II). }\end{array}$ \\
\hline Food & $\begin{array}{l}\text { Chimpanzees share food with friends but not with nonfriends (Engelmann and } \\
\text { Herrmann 20I6). }\end{array}$ \\
\hline Copulation rules & $\begin{array}{l}\text { Juvenile chimpanzee males who venture too close to an estrus female risk being } \\
\text { attacked by adult males (de Waal 20I4) }\end{array}$ \\
\hline $\begin{array}{l}\text { Immigrant } \\
\text { conformity }\end{array}$ & $\begin{array}{l}\text { Immigrant chimpanzees modify their tool use to conform to the practices of } \\
\text { their new community, even though the adopted practice is less functional } \\
\text { (Luncz et al., 20I 2; Luncz and Boesch 20I4) }\end{array}$ \\
\hline $\begin{array}{l}\text { Arbitrary } \\
\text { conventions }\end{array}$ & $\begin{array}{l}\text { A female chimpanzee started wearing a straw-like blade of grass in her ear, and } \\
\text { other chimpanzees began to do the same (van Leeuwen, Cronin, and Haun } \\
\text { 20I4); chimpanzees prefer to open a puzzle box in the way demonstrated by } \\
\text { higher-ranking group members (Horner et al. 2006). }\end{array}$ \\
\hline Inequity avoidance & $\begin{array}{l}\text { Chimpanzees refuse to participate in tasks upon witnessing another receive a } \\
\text { higher-valued reward (Brosnan, Schiff, and de Waal 2005, Brosnan et al. } \\
\text { 20I0; de Waal and Brosnan 2003). Chimpanzees in an ultimatum game } \\
\text { make more equitable divisions after partner protests (Proctor et al. 20I3). }\end{array}$ \\
\hline Cooperation & $\begin{array}{l}\text { Chimpanzees work together to achieve a joint goal, including cooperative } \\
\text { hunting in wild chimpanzees (Boesch I994); captive chimpanzees } \\
\text { coordinate rope pulling to access shared food (Crawford I937; Hirata and } \\
\text { Fuwa 2007); captive chimpanzees choose to work together using an } \\
\text { apparatus available to a group of eleven chimpanzees, mitigating } \\
\text { competition (Suchak et al. 20I6) }\end{array}$ \\
\hline Consolation & $\begin{array}{l}\text { Chimpanzees engage in higher levels of affiliation with a social partner after a } \\
\text { conflict and facilitate reconciliation between fighting parties (Kutsukake } \\
\text { and Castles 2004; de Waal 2009). }\end{array}$ \\
\hline In-group preference & $\begin{array}{l}\text { Chimpanzees patrol boundaries between neighboring communities, } \\
\text { sometimes invading and killing adult males and infants and kidnapping } \\
\text { adult females (Watts and Mitani 200I; Watts et al. 2006) }\end{array}$ \\
\hline $\begin{array}{l}\text { Highly respected } \\
\text { elders }\end{array}$ & $\begin{array}{l}\text { Elderly males have a special status in the community, even though they are } \\
\text { often low ranking. Group members defer to elders when deciding which } \\
\text { direction to travel, share meat with elders, and fight over who gets to groom } \\
\text { an elder (Nishida 20I2). }\end{array}$ \\
\hline Weaning & $\begin{array}{l}\text { Weaning begins around age four and can last for more than a year. Weanlings } \\
\text { manipulate their mother into giving them access to milk by engaging in } \\
\text { dangerous behavior, such as approaching older males or human observers } \\
\text { (Nishida 2OI } 2 \text { ). }\end{array}$ \\
\hline
\end{tabular}

of infanticide than at videos showing monkey hunting, nut cracking, or male dominance displays, but they did not demonstrate any emotional response to these videos (Rudolf von Rohr et al. 20I5). More research along these lines can help to uncover the cause of the longer looking pattern. Field research that seeks to examine long-term responses to candidate norm violators, such as avoiding or 
shunning a violator, expressing negative emotions to the violator, making specific vocalizations in response to a violation, or even facilitating a restoration of a relationship between two others, could serve as another source of evidence. For example, researchers report third-party reconciliation between members of M-group in Mahale (Kutsukake and Castles 2004).

Naïve normativity in chimpanzees, like naïve normativity in children, might be early developing in infancy, but more research is required to evaluate this hypothesis. That we see preliminary evidence of naïve normativity in adult ape social interaction, however, supports the hypothesis that naive normativity might be an ancient human cognitive endowment that is more widespread in other species than typically thought.

\section{Conclusion}

Whether animals are moral agents or have moral cognition is going to be dependent on one's view about the nature of morality. Even some of the strongest supporters of morality in animals, such as Frans de Waal (2006; 20I4), speak only of proto-norms or proto-moral agency in apes. To make progress on the topic of the evolution of morality, we need to identify and clearly define the elements that make up moral practice, rather than relying on a vague sense of proto-morality. At this point, it is of more benefit to examine the evolution of normative practice.

If I am right, then normative thinking is implicit in human social cognition. Furthermore, because the empirical evidence suggests that both apes and human beings take others to be intentional agents-some of whom are in-group members and good sources of normative information and sanctioned when they act against expectations-we have preliminary evidence of normative cognition in other apes.

To find additional evidence for animal social norms, we should look for them in the places we would expect to find them, that is in free-living wild apes or long-term captive communities where established norms have had time to evolve. Since norms are cultural items, rather than biological ones, when we take chimpanzees from different cultures and put them together to create a new group, we disrupt normative practices. Fieldwork is slow and can be tedious, but fieldwork in unfamiliar places is needed to answer our questions about the evolution of normative thinking.

KRISTIN ANDREWS

YORK UNIVERSITY

andrewsk@yorku.ca

\section{References}

Allen, Jedediah W. P., and Mark H. Bickhard. (20II) 'Emergent Constructivism'. Child Development Perspectives, 5, I64-65.

Andrews, Kristin. (20I2) Do Apes Read Minds? Toward a New Folk Psychology. Cambridge, MA: MIT Press.

Andrews, Kristin. (2015) 'The Folk Psychological Spiral: Explanation, Regulation, and Language'. Southern Journal of Philosophy, 53, 50-67. 
Banerjee, Robin, Mark Bennett, and Nikki Luke. (20I0) 'Children's Reasoning about the Self-Presentational Consequences of Apologies and Excuses Following Rule Violations'. British Journal of Developmental Psychology, 28, 799-8 I 5.

Bian, Lin, Stephanie Sloane, and Renée Baillargeon. (2018) 'Infants Expect Ingroup Support to Override Fairness When Resources Are Limited'. PNAS, I I 5(II), 2705-IO.

Bicchieri, Cristina. (2017) Norms in the Wild: How to Diagnose, Measure, and Change Social Norms. New York: Oxford University Press.

Boesch, Christophe. (I994) 'Cooperative Hunting in Wild Chimpanzees'. Animal Behavior, 48, 653-67. Braithwaite, John. (2002) Restorative Justice and Responsive Regulation. New York: Oxford University Press.

Brosnan, Sarah F., Catherine Talbot, Megan Ahlgren, Susan P. Lambeth, Stephen J. Shapiro. (20I0) 'Mechanisms Underlying Responses to Inequitable Outcomes in Chimpanzees, Pan troglodytes'. Animal Behaviour, 79, I229-37.

Brosnan, Sarah F., Hillary C. Schiff, and Frans B. M. de Waal. (2005) 'Tolerance for Inequity May Increase with Social Closeness in Chimpanzees'. Proceedings of the Royal Society B: Biological Sciences, 272, 253-58.

Buttelmann, David, Norbert Zmyj, Mortiz Daum, and Malinda Carpenter. (20I3) 'Selective Imitation of In-Group over Out-Group Members in I4-Month-Old Infants'. Child Development, 84, 422-28.

Byrne, Richard W., and Anne E. Russon. (I998) 'Learning by Imitation: A Hierarchical Approach'. Behavioral and Brain Sciences, 2I, 667-84.

Crawford, Meredith P. (1937) The Coöperative Solving of Problems by Young Chimpanzees. Baltimore: Johns Hopkins Press.

Engelmann, Jan M., and Esther Herrmann. (20I6) 'Chimpanzees Trust Their Friends'. Current Biology, 26, 252-6.

Fitzpatrick, Simon. (2017) 'Animal Morality: What Is the Debate About?' Biology and Philosophy, 32, II 5 I -83 .

Fraser, Orlaith N., Daniel Stahl, and Filippo Aureli. (2008) 'Stress Reduction through Consolation in Chimpanzees'. PNAS, 105, 8557-62.

Gergely, György, Zoltán Nádasdy, Csibra Gergely, and Szilvia Bíró. (1995) ‘Taking the Intentional Stance at I 2 Months of Age'. Cognition, 56, I65-93.

Gergely, György, Harold Bekkering, and Ildikó Király. (2002) 'Rational Imitation in Preverbal Infants'. Nature, 4I 5, 755 .

Goodall, Jane. (I986) 'Social Rejection, Exclusion, and Shunning among the Gombe Chimpanzees'. Ethology and Sociobiology, 7, 227-36.

Henrich, Joseph. (2017) The Secret of Our Success: How Culture Is Driving Human Evolution, Domesticating Our Species, and Making Us Smarter. Princeton, NJ: Princeton University Press.

Hirata, Satoshi, and Kohki Fuwa. (2007) 'Chimpanzees (Pan troglodytes) Learn to Act with Other Individuals in a Cooperative Task'. Primates, $48, \mathrm{I}_{3}-2 \mathrm{I}$.

Hockings, Kimberly J., James R. Anderson, and Tetsuro Matsuzawa. (2006) 'Road Crossing in Chimpanzees: A Risky Business'. Current Biology, I6, R668-70.

Hopper, Lydia M., Antoine Spiteri, Susan P. Lambeth, Steven J. Schapiro, Victoria Horner, and Andrew Whiten. (2007) 'Experimental Studies of Traditions and Underlying Transmission Processes in Chimpanzees'. Animal Behaviour, 73, I02I-32.

Hopper, Lydia M., Susan P. Lambeth, Steven J. Schapiro, and Whiten, A. (2008) 'Observational Learning in Chimpanzees and Children Studied through "Ghost" Conditions'. Proceedings of the Royal Society of London B: Biological Sciences, 275(I636), 835-40.

Horner, Victoria, and Andrew Whiten. (2004) 'Causal Knowledge and Imitation/Emulation Switching in Chimpanzees (Pan troglodytes) and Children (Homo sapiens)'. Animal Cognition, 8, I64-8I.

Horner, Victoria, Andrew Whiten, Emma Flynn, and Frans B. M. de Waal. (2006) 'Faithful Copying of Foraging Techniques along Cultural Transmission Chains by Chimpanzees and Children'. PNAS, 103, I3878-83.

Jensen, Keith, Josep Call, and Michael Tomasello. (2007) 'Chimpanzees are Vengeful but not Spiteful'. PNAS, 104, I3046-50. 
Jin, Kyong-sun, and Renée Baillargeon. (20I7) 'Infants Possess an Abstract Expectation of Ingroup Support'. PNAS, II4(3I), 8I99-8204.

Kahlenberg, Sonya M., Melissa Emory Thompson, Martin N. Muller, and Richard W. Wrangham. (2008) 'Immigration Costs for Female Chimpanzees and Male Protection as an Immigrant Counterstrategy to Intrasexual Aggression'. Animal Behaviour, 76, I497-509.

Kelly, David J., Paul C. Quinn, Alan M. Slater, Kang Lee, Alan Gibson, Michael Smith, Liezhong Ge, and Olivier Pascalis. (2005) 'Three-Month-Olds, But Not Newborns, Prefer Own-Race Faces'. Developmental Science, 8, F3 I-36, 459-620.

Kendal, Rachel, Lydia M. Hopper, Andrew Whiten, Sarah F. Brosnan, Susan P. Lambeth, Steven J. Schapiro, and Will Hoppitt. (2015) 'Chimpanzees Copy Dominant and Knowledgeable Individuals: Implications for Cultural Diversity'. Evolution and Human Behavior, 36, 65-72.

Kinzler Katherine, D., Emmanuel Dupoux, and Elizabeth S. Spelke. (2007) 'The Native Language of Social Cognition'. PNAS, I04, I2577-80.

Kitcher, Philip. (20I I) The Ethical Project. Cambridge, MA: Harvard University Press.

Korsgaard, Christine. (2018) Fellow Creatures: Our Obligations to the Other Animals. Oxford: Oxford University Press.

Kutsukake, Nobuyuki, and Duncan L. Castles. (2004) 'Reconciliation and Post-conflict Third-Party Affiliation among Wild Chimpanzees in the Mahale Mountains, Tanzania'. Primates, 45, I 57-65.

van Leeuwen, Edwin J. C., Katherine A. Cronin, and Daniel B. M. Haun. (2014) 'A Group-Specific Arbitrary Tradition in Chimpanzees (Pan troglodytes)'. Animal Cognition, I7, I42 I-25.

Liberman, Zoe, Katherine D. Kinzler, and Amanda L. Woodward. (20I4) 'Friends or Foes: Infants Use Shared Evaluations to Infer Others' Social Relationships'. Journal of Experimental Psychology: General, I43, 966-7I.

Luncz, Lydia V., and Boesch, Christophe. (20I4) 'Tradition over Trend: Neighboring Chimpanzee Communities Maintain Differences in Cultural Behavior despite Frequent Immigration of Adult Females'. American Journal of Primatology, 76, 649-57.

Luncz, Lydia V., Mundry, Roger, and Boesch, Christophe. (20I2) 'Evidence for Cultural Differences between Neighboring Chimpanzee Communities'. Current Biology, 22, 922-26.

Lyons, Derek E., Andrew G. Young, and Frank C. Keil. (2007). 'The Hidden Structure of Overimitation'. Proceedings of the National Academy Sciences, I04, I975 I-56.

Mahajan, Neha, and Karen Wynn. (2012) 'Origins of "Us" versus "Them": Prelinguistic Infants Prefer Similar Others'. Cognition, I 24, 227-33.

Maibom, Heidi L. (2007) 'Social Systems'. Philosophical Psychology, 20, 557-78.

McGeer, Victoria. (2007) 'The Regulative Dimension of Folk Psychology'. In Daniel D. Hutto and Matthew Ratcliffe (eds.), Folk Psychology Re-Assessed (Dordrecht: Springer), I37-56.

McGeer, Victoria. (2015) 'Mind-Making Practices: The Social Infrastructure of Self-Knowing Agency and Responsibility'. Philosophical Explorations, I 8, 259-8 I.

Meltzoff, Andrew N. (I988) 'Infant Imitation after a I-Week Delay: Long-Term Memory for Novel Acts and Multiple Stimuli'. Developmental Psychology, 24, 470-76.

Mendes, Natacha, Nikolaus Steinbeis, Nereida Bueno-Guerra, Josep Call, and Tania Singer. (20I8) 'Preschool Children and Chimpanzees Incur Costs to Watch Punishment of Antisocial Others'. Nature Human Behaviour, 2, 45-5I.

Monsó, Susana. (2015) 'Empathy and Morality in Behaviour Readers'. Biology and Philosophy, 30 , $67 \mathrm{I}-90$.

Monsó, Susana and Kristin Andrews. (forthcoming) 'Animal Moral Psychologies'. In John M. Doris and Manuel Vargas (eds.), The Moral Psychology Handbook (second edition) (Oxford: Oxford University Press).

Murray, Carson M., Emily Wroblewski, and Anne E. Pusey. (2007) 'New Case of Intragroup Infanticide in the Chimpanzees of Gombe National Park'. International Journal of Primatology, 28, 23-37.

Myowa-Yamakoshi, Masako, and Tetsuro Matsuzawa. (2000) 'Imitation of Intentional Manipulatory Actions in Chimpanzees (Pan troglodytes)'. Journal of Comparative Psychology, I I 4, 38I-39I.

Nishida, Toshisada. (20I2) Chimpanzees of the Lakeshore: Natural History and Culture at Mahale. Cambridge: Cambridge University Press. 
Nucci, Larry P., and Elliot Turiel. (1978) 'Social Interactions and the Development of Social Concepts in Preschool Children'. Child Development, 49, 400-7.

Oakes, Lisa M. (20I0) 'Using Habituation of Looking Time to Assess Mental Processes in Infancy'. Journal of Cognition and Development, I I, 25 5-68.

Ohashi, Gaku, and Tetsuro Matsuzawa. (20II) 'Deactivation of Snares by Wild Chimpanzees'. Primates, 52, I-5.

Proctor, Darby, Rebecca A. Williamson, Frans B. M. de Waal, and Sarah F. Brosnan. (2013) 'Chimpanzees Play the Ultimatum Game'. PNAS, i Iо, 2070-75.

Pun, Anthea, Matar Ferera, Gil Diesendruck, J. Kiley Hamlin, and Andrew S. Baron. (2018) 'Foundations of Infants' Social Group Evaluations'. Developmental Science, 21, eI 2586.

Rakoczy, Hannes, Felix Warneken, and Michael Tomasello. (2008) 'The Sources of Normativity: Young Children's Awareness of the Normative Structure of Games'. Developmental Psychology, 44, 875-8I.

Rakoczy, Hannes, Felix Warneken, and Michael Tomasello. (2009)' 'Young Children's Selective Learning of Rule Games from Reliable and Unreliable Models'. Cognitive Development, 24, 6I-9.

Richerson, Peter J., and Robert Boyd. (2005) Not By Genes Alone: How Culture Transformed Human Cognition. Chicago: University of Chicago.

Riedl, Katrin, Keith Jensen, Josep Call, and Michael Tomasello. (20I 2) 'No Third-Party Punishment in Chimpanzees'. PNAS, 109, I4824-29.

Rowlands, Mark. (2012) Can Animals Be Moral? New York: Oxford University Press.

Rudolf von Rohr, Claudia, Sonja E. Koski, Judith M. Burkart, Clare Caws, Orlaith N. Fraser, Angela Ziltener, and Carel P. van Schaik. (20I2) 'Impartial Third-Party Interventions in Captive Chimpanzees: A Reflection of Community Concern'. PLOS ONE 7, e32494.

Rudolf von Rohr, Claudia, Carel P. van Schaik, Alexandra Kissling, and Judith M. Burkart. (2015) 'Chimpanzees' Bystander Reactions to Infanticide'. Human Nature, 26, I43-60.

Sterelny, Kim. (20I2) The Evolved Apprentice: How Evolution Made Humans Unique. Cambridge, MA: MIT Press.

Suchak, Malini, Timothy M. Eppley, Matthew W. Campbell, Rebecca A Feldman, Luke F. Quarles, and Frans B. M. de Waal. (2016) 'How Chimpanzees Cooperate in a Competitive World'. PNAS, II3, IO2I 5-2O.

Suddendorf, Thomas. (2013) The Gap: The Science of What Separates Us from Other Animals. New York: Basic Books.

Tennie, Claudio, Josep Call, and Michael Tomasello. (2006) 'Push or Pull: Imitation vs. Emulation in Great Apes and Human Children'. Ethology, I I 2, I I 59-69.

Tomasello, Michael. (1999) The Cultural Origins of Human Cognition. Cambridge, MA: Harvard University Press.

Tomasello, Michael. (20I4) A Natural History of Human Thinking. Cambridge, MA: Harvard University Press.

Uller, Claudia. (2004) 'Disposition to Recognize Goals in Infant Chimpanzees'. Animal Cognition, 7 , I 54-6I.

Vincent, Sarah, Rebecca Ring, and Kristin Andrews. (2019) 'Normative Practices of Other Animals'. In Aaron Zimmerman, Karen Jones, and Mark Timmons (eds.), Routledge Handbook of Moral Epistemology (New York: Routledge), 57-83.

de Waal, Frans B. M. (I982) Chimpanzee Politics: Power and Sex among Apes. London: Jonathan Cape.

de Waal, Frans B. M. (2006) 'The Tower of Morality'. In Josiah Ober and Stephen Macedo (eds.), Primates and Philosophers: How Morality Evolved (Princeton: Princeton University Press), I6I-8I.

de Waal, Frans B. M. (2009) The Age of Empathy: Nature's Lessons for a Kinder Society. Toronto: McClelland \& Stewart.

de Waal, Frans B. M. (20I4) 'Natural Normativity: The "Is" and "Ought” of Animal Behaviour'. Behaviour, I5I, I 85-204.

de Waal, Frans B. M., and Sarah F. Brosnan. (2003) 'Monkeys Reject Unequal Pay'. Nature, 425, 297. 
Watts, David P., John C. Mitani. (200I) 'Boundary Patrols and Intergroup Encounters in Wild Chimpanzees'. Behaviour, I38, 299-327.

Watts, David P., Martin Muller, Sylvia Amsler, Godfrey Mbabazi, and John C. Mitani. (2006) 'Lethal Intergroup Aggression by Chimpanzees in Kibale National Park, Uganda'. American Journal of Primatology, 68, I6I-80.

Webb, Christine E., Teresa Romero, Becca Franks, and Frans B. M. de Waal. (20I7) 'Long-Term Consistency in Chimpanzee Consolation Behaviour Reflects Empathetic Personalities'. Nature Communications, 8, Article 292.

Whiten, Andrew, Nicola McGuigan, Sarah Marshall-Pescini, and Lydia M. Hopper. (2009) 'Emulation, Imitation, Over-Imitation and the Scope of Culture for Child and Chimpanzee'. Philosophical Transactions of the Royal Society of London B: Biological Sciences, 364, 24 I7-28.

Whiten, Andrew, Antoine Spiteri, Victoria Horner, Kristen Bonnie, Susan P. Lambeth, Steven J. Schapiro, and Frans B. M. de Waal. (2007) 'Transmission of Multiple Traditions within and between Chimpanzee Groups'. Current Biology, I7, I038-43.

Wiessner, Polly, and Nitze Pupu. (2O I 2) 'Toward Peace: Indigenous Institutions and Foreign Arms in a Papua New Guinea Society'. Science, 337, I65 I-54.

Woodward, Amanda L. (I999) 'Infants' Ability to Distinguish between Purposeful and Non-purposeful Behaviors'. Infant Behavior and Development, 22, I45-60.

Xiao, Naiqi G., Rachel Wu, Paul C. Quinn, Shaoying Liu, Kristen S. Tummeltshammer, Natasha Z. Kirkham, Liezhong Ge, Olivier Pascalis, and Kang Lee. (20I8) 'Infants Rely More on Gaze Cues from Own-Race than Other-Race Adults for Learning under Uncertainty'. Child Development, 89, e229-44.

Yamamoto, Shinya, Tatyana Humle, and Masayuki Tanaka. (2009) 'Chimpanzees Help Each Other Upon Request'. PLoS ONE, 4, e74I6.

Zawidzki, Tadeusz Wiesław. (2013) Mindshaping: A New Framework for Understanding Human Social Cognition. Cambridge, MA: MIT Press. 